\title{
CRESCIMENTO E DISTRIBUIÇÃO RADICIAL DE TRÊS CULTIVARES \\ DE CANA-DE-AÇÚCAR, EM CANA SOCA, EM DOIS TIPOS \\ DE SOLO, EM RIZOTRON. I. USO DO WinRHIZO'
}

\section{ROOT GROWTH AND DISTRIBUTION OF THREE SUGARCANE CULTIVARS, IN RATOON CROP, IN TWO SOIL TYPES, IN RHIZOTRON. I. USE OF WIRRHIZO}

\author{
Oswaldo Teruyo IDO² \\ Edelclaiton DAROS ${ }^{3}$ \\ José Luis Camargo ZAMBON ${ }^{3}$ \\ Heroldo WEBER ${ }^{4}$ \\ Pedro Henrique de Medeiros BUSO ${ }^{5}$ \\ Ricardo Augusto de OLIVEIRA ${ }^{5}$
}

\begin{abstract}
RESUMO
O presente trabalho teve como objetivo avaliar o crescimento e a distribuição do sistema radicial, em cana de primeira soca, de três cultivares de cana-de-açúcar, em dois tipos de substrato (Argiloso e Arenoso), em condições de rizotron. A pesquisa foi realizada na Estação Experimental de Paranavaí/SCA/UFPR, de junho de 2000 a julho de 2001. Analisaram-se o comprimento e a distribuição das raízes através da metodologia de amostras volumétricas associadas às leituras do WinRHIZO. O substrato influenciou o crescimento radicial das cultivares. Quanto à distribuição quantitativa do comprimento total das raízes as RB835486 e SP80-1842 apresentaram melhores desempenhos no substrato argiloso, enquanto a cultivar RB855536 mostrou maior quantidade do sistema radicial no substrato arenoso. O rizotron é uma estrutura viável para o estudo do sistema radicial da cana soca.
\end{abstract}

Palavras-chave: sistema radicial, estudo de raízes, desenvolvimento.

\begin{abstract}
The present work had as objective to evaluate the growth and the distribution of the root system, in the first ratoon crop cycle, of three sugarcane cultivars, in two substratum types (Loamy and Sandy), in rhizotron conditions. The research was accomplished in the Experimental Station of Paranavaí/SCA/UFPR, of June of 2000 to July of 2001 . The root length and the distribution were analyzed through the methodology of samples volumetric as associated to the readings of WinRHIZO. The substratum influenced the root growth of the cultivars. With relationship to the quantitative distribution of the length total of the roots RB835486 and SP80-1842 presented better performances in the loamy substratum, while the cultivar RB855536 showed larger amount of the root system in the sandy substratum. The rhizotron is a viable structure for the study of the root system of the ratoon crop.
\end{abstract}

Key-words: root system, study of roots, development.

\footnotetext{
${ }^{1}$ Parte do trabalho de Tese de Doutorado do primeiro autor. correspondência <ido@ufpr.br>.

${ }^{3} \mathrm{Eng}^{\circ}$ Agr', D.Sc., Professor Adjunto do Curso de Agronomia da UFPR.

${ }^{4}$ Eng $^{\circ}$ Agr', D Sc., Pesquisador, DFF/SCA/UFPR.

${ }^{5} \mathrm{Eng}^{\circ}$ Agr', Aluno de Pós-graduação em Agronomia, Produção Vegetal, UFPR.
}

${ }^{2}$ Eng $^{\circ}$ Agr ${ }^{\circ}$, D.Sc., Professor Adjunto do Curso de Agronomia, UFPR. Rua dos Funcionários, 1540, Juvevê, CEP: 80035-050, Curitiba, PR. Autor para 


\section{INTRODUÇÃO}

Os estudos do sistema radicial da cana-deaçúcar em rizotron são ainda escassos. Wei e Yang (1987) citam que o primeiro laboratório para estudos de raiz da cana-de-açúcar foi construído em Mount Edgecomb na África do Sul.

Segundo Matta (1999), de maneira geral a gama de informações sobre o desenvolvimento da raiz é exígua, comparativamente, perto do que se sabe sobre a parte aérea, e se pouco ainda se conhece sobre os mecanismos de desenvolvimento do caule, muito menos se sabe sobre os da raiz. Portanto, é indiscutível a necessidade de mais informações sobre o desenvolvimento e distribuição de raízes de plantas em diferentes solos e diversas condições ecológicas (VASCONCELOS et al.,1999).

Vários trabalhos têm demonstrado a importância do estudo do sistema de raízes de diferentes plantas cultivadas, apresentando também várias metodologias para caracterização de raízes, levando em conta custo, precisão e tempo de análise (JORGE, 1999).

O objetivo deste trabalho foi o de avaliar o crescimento e a distribuição do sistema radicial de três cultivares de cana-de-açúcar, em cana de primeira soca, em dois tipos de solo, em condições de rizotron, usando a metodologia do WinRHIZO.

\section{MATERIAL E MÉTODOS}

O experimento foi instalado na Estação Experimental de Cana-de-Açúcar, da UFPR, em Paranavaí-PR, em condições de rizotron (DAROS et al.,1999). O rizotron tem $2,05 \mathrm{~m}$ de altura, 7,0 $\mathrm{m}$ de comprimento, sendo construído em tronco trapézio com 4,22 $\mathrm{m}$ de largura na base inferior e 2,20 m na parte superior, observando a inclinação de $25^{\circ}$. A parede interna é constituída de vidro temperado transparente de 12 milímetros com 1,00 m x 2,20 m e a externa com placas de cimento amianto de 4 milímetros. O espaço entre o vidro e a placa de amianto é regulável e pode ir até a $30 \mathrm{~cm}$, onde se coloca o substrato. Este, foi dividido em quatorze subdivisões (janelas) de 1,00 m de largura por 0,25 $\mathrm{m}$ de comprimento por 2,20 $\mathrm{m}$ de profundidade.

Os solos utilizados como substratos foram coletados em Paranavaí (arenoso - Latossolo Vermelho Distrófico Típico A moderado textura média), e em Bandeirantes (argiloso - Latossolo Vermelho Eutroférrico A moderado textura argilosa) (EMBRAPA, 1999). Suas características químicas e físicas são apresentadas na Tabela 1. Em função dos teores encontrados nas análises, os substratos foram homogeneizados com fertilizantes, elementos simples, para elevar a saturação de potássio a $5 \%$ da CTC, fósforo a $60 \mathrm{mg}^{\mathrm{dm}} \mathrm{d}^{-3}$, mais $1 \mathrm{t}$ de calcário (PRNT = 100) e nitrogênio, aplicado na proporção de 100 $\mathrm{kg} \cdot \mathrm{ha}^{-1}$.

O plantio foi realizado em 06 de abril de 1999, colocando-se um tolete de uma gema pré-germinada por janela. As mudas vieram de viveiros da Estação
Experimental de Paranavaí com 11 meses de idade. A colheita da cana planta foi no dia 27 de junho de 2000 , sendo em seguida realizada à adubação de cana soca com o equivalente de $20-00-20 \mathrm{com} 600 \mathrm{~kg} \mathrm{ha}^{-1}$. A colheita de cana soca ocorreu no dia 10 de julho de 2001. A umidade do substrato foi mantida próxima a capacidade de campo ao longo do perfil por meio de irrigação suplementar por gotejo. As cultivares em estudo foram: RB835486, RB855536 e SP80-1842.

Para avaliação das raízes foram feitas amostragens em cada subdivisão (janela), após a retirada das placas externas de amianto, em nove profundidades $(20,40,60,80,100,120,140,160$, e $180 \mathrm{~cm}$ ), por ocasião da colheita da cana planta (0); $56,92,126,161,210,252,316$ e 378 dias após o corte da cana planta (DAC), utilizando cilindro volumétrico com $20 \mathrm{~cm}$ de altura, diâmetro interno de $4,5 \mathrm{~cm}$ e parede externa de $0,5 \mathrm{~cm}$, com borda serrilhada e cortante numa das extremidades (volume de $318 \mathrm{~cm}^{3}$ ). Evitando sobreposição de coleta em cada avaliação, alternadamente foi amostrado lado esquerdo ou direito, ao redor de $20 \mathrm{~cm}$ distantes da linha central da touceira.

As porções de solo e raiz das amostras foram deixadas em $25 \mathrm{ml}$ de hidróxido de sódio $1 \mathrm{~N}$ diluído em um litro de água durante 12 horas para a dispersão da argila, e em seguida lavagem em água corrente sobre jogo de peneiras com malhas de 0,50 e $0,25 \mathrm{~mm}$. A separação das raízes da canade-açúcar de outros materiais minerais e orgânicos foi realizada manualmente com pinças de ponta fina em água. As raízes foram acondicionadas em potes plásticos contendo solução de etanol $50 \%$ e armazenados a $0^{\circ} \mathrm{C}$ conforme Böhm (1979) até determinação dos seus parâmetros.

Os dados de comprimento de raízes foram obtidos no Laboratório de Fitotecnia, do Departamento de Fitotecnia e Fitossanitarismo do SCA/UFPR, em Curitiba-PR, por um sistema de análise de imagens, o RHIZO, versão 4.1c para ambiente Windows (RÉGENT INSTRUMENTS, 1999 a e b). Todas as avaliações foram realizadas com nível de resolução médio de 200 dpi. Na colheita, os colmos foram colhidos rente ao substrato, fazendo o desponte (fixado sempre no colarinho da folha +5$)$. Após secagem em estufa com circulação de ar forçada até peso constante, foram determinadas a massa seca total da parte aérea (folhas + bainhas + palmito + colmos).

\section{RESULTADOS E DISCUSSÃO}

Nas Tabelas 2, 3, 4 estão os resultados das avaliações do comprimento total das raízes, nos períodos de desenvolvimento da cana de primeira soca, em condições de rizotron.

Considerando a soma total das nove amostragens realizadas (Tabela 2), a cultivar RB835486 apresentou no substrato argiloso uma quantidade total de comprimento de raízes de 1,323 m. $0,002862 \mathrm{~m}^{-3}$ de solo contra $1,223 \mathrm{~m}$ no substrato arenoso, com uma diferença superior de $8,2 \%$.

$\mathrm{Na}$ colheita da cana de primeira soca (378 
DAC) ocorreu no substrato argiloso uma tendência de aumento no comprimento radicial (183,8 $\mathrm{m} .0,002862 \mathrm{~m}^{-3}$ de solo), ao contrário do substrato arenoso, que apresentou tendência de decréscimo no comprimento total de raízes $\left(129,7 \mathrm{~m} .0,002862 \mathrm{~m}^{-3}\right.$ de solo), indicando que a cultivar RB835486 possui um sistema radicial com capacidade de adaptação tanto em solo arenoso como argiloso, aumentando a produção radicial no substrato argiloso tanto em quantidade como em profundidade, explorando e utilizando melhor este substrato para melhor aporte necessário para a produção de massa seca aérea total por ocasião da colheita de 16,05 kg/janela, bem como, no substrato arenoso diminuindo a produção de raízes para mobilizar a distribuição dos fotoassimilados para a formação e sustentação da massa seca aérea total (12,06 kg/janela).

Portanto, a diferença ocorrida na produção total radicial, na cana de primeira soca foi em função das melhores condições físicas do substrato argiloso. Este fato pode ser justificado por VITTI e MAZZA (2002), que comentam que um substrato que apresenta menor velocidade de drenagem pode proporcionar à cultura da cana-de-açúcar melhor aproveitamento da água, assim como aumentar a eficiência de aproveitamento de alguns nutrientes, principalmente nitrogênio e potássio, altamente lixiviáveis através da drenagem.

A cultivar RB855536, considerada de excelente brotação de soca (ARIZONO et al., 2000), independente do tipo de substrato, por ocasião da brotação da soqueira apresenta aumento na produção de raízes nos primeiros $60 \mathrm{DAC}$, com distribuição das raízes ao longo do perfil dos substratos, com leve tendência de concentração nas camadas superficiais de 0-60 cm, tanto no substrato argiloso de $36 \%$ como no substrato arenoso de $38 \%$ (Tabela 3). Na colheita de julho de 2001, o comprimento de raízes no substrato arenoso $(210,5 \mathrm{~m})$ foi de 1,15 vezes maiores do que no substrato argiloso (182,6 m).

Os aumentos nas quantidades do sistema radicial no substrato arenoso não proporcionaram aumento na produtividade final da parte aérea, pois a massa seca aérea total por ocasião da colheita da cana de primeira soca, no compartimento do substrato argiloso $(14,32 \mathrm{~kg} / \mathrm{janela})$ foi superior ao substrato arenoso (8,99 kg/janela) na ordem de 1,59 vezes, o que pode ser justificado segundo Taylor (1981), Matsuoka (1996) e Vasconcelos (2002), que consideram que a maior massa radicial pode significar perda de produtividade, pois parte das reservas, que poderiam ser utilizada para a produção de colmos e folhas, é drenada para o aumento e manutenção do sistema radicial.

$\mathrm{Na}$ média geral, a cultivar RB855536 apresentou uma distribuição percentual no comprimento de raízes, no substrato arenoso de $36 \%$ e no argiloso $35 \%$ nos primeiros $60 \mathrm{~cm}$ superficiais e o restante da quantidade de raízes distribuídas uniformemente ao longo do perfil dos substratos.

A cultivar SP80-1842 apresenta por ocasião da colheita da cana planta um comprimento total das raízes no substrato argiloso de 137,5 m.0,002862 $\mathrm{m}^{-3}$ de solo, sendo 1,33 vezes mais do que $103,7 \mathrm{~m}$ do substrato arenoso (Tabela 4). Semelhantemente na colheita da cana de primeira soca (378 DAC), também no substrato argiloso $\left(207,5 \mathrm{~m} .0,002862 \mathrm{~m}^{-3}\right.$ de solo) foi superior no comprimento total de raízes em 1,40 vezes em relação ao substrato arenoso (148,6 m). Em termos de quantidade total de comprimento radicial no substrato argiloso foi de 1,17 vezes maiores quando comparado ao substrato arenoso.

$\mathrm{Na}$ cana de primeira soca, os aumentos nas quantidades do sistema radicial no substrato argiloso, propiciaram aumento de 1,23 vezes mais massa seca aérea total, por ocasião da colheita, no substrato argiloso (14,83 kg/janela) do que no substrato arenoso $(12,02 \mathrm{~kg} / \mathrm{janela})$, o que pode ser explicado, em função da melhor distribuição radicial (maior comprimento) proporcionaram maior eficiência na exploração do substrato, somado a melhor condição física do substrato argiloso, o que está de acordo com KORNDÖRFER et al. (1989), que citam que quanto maior o sistema radicial de uma planta maior sua capacidade de explorar o solo e consequentemente aproveitar os nutrientes e a água disponível. O volume e a distribuição do sistema radicial são tanto mais importantes quanto menor a fertilidade do solo e maior a deficiência hídrica. Estes resultados indicam uma tendência de exigência em fertilidade de solo em cana soca da cultivar SP80-1842.

TABELA 1 - Resultados das análises químicas e granulometria do substrato (A), argiloso, e do substrato (B), arenoso.

\begin{tabular}{|c|c|c|c|c|c|c|c|c|c|c|c|}
\hline & \multicolumn{8}{|c|}{ Química } & \multicolumn{3}{|c|}{ Física } \\
\hline & $\mathrm{pH}$ & $\mathrm{Al}^{3-}$ & $\mathrm{H}+\mathrm{Al}$ & $\mathrm{Ca}^{2}+$ & $\mathrm{Mg}^{2+}$ & $\mathrm{K}^{+}$ & $P$ & C & Areia & Silte & Argila \\
\hline (Camada) & $\mathrm{CaC}_{2}$ & \multicolumn{5}{|c|}{$\mathrm{Cmol}_{\mathrm{c}} \cdot \mathrm{dm}^{-3}$} & Mg.dm ${ }^{-3}$ & g.dm ${ }^{-3}$ & \multicolumn{3}{|c|}{$\%$} \\
\hline & & & & & trato & (Arg & & & & & \\
\hline $0-20 \mathrm{~cm}$ & 5,0 & 0,0 & 5,45 & $\begin{array}{r}5,20 \\
\text { St }\end{array}$ & $\begin{array}{l}1,60 \\
\text { trato }\end{array}$ & $\begin{array}{l}0,16 \\
\text { (Arer }\end{array}$ & ) & 26,8 & 9,0 & 22 & 69 \\
\hline $0-20 \mathrm{~cm}$ & 5,2 & 0,0 & 2,37 & 1,15 & 0,85 & 0,15 & 8,0 & 9,19 & 82 & 1,0 & 17 \\
\hline
\end{tabular}


IDO, O.T. et al. Crescimento e distribuição radical...

TABELA 2 - Distribuição quantitativa e percentual de comprimento $\left(\mathrm{m} \mathrm{0,000318} \mathrm{m}^{-3}\right)$ de raízes da cultivar RB835486, em cana relação à profundidade, em RIZOTRON, através de nove amostragens com cilindro volumétrico, em extratos de substratos (argiloso e arenoso), Estação Experimental de Paranavaí/PR - UFPR, JUL/2001.

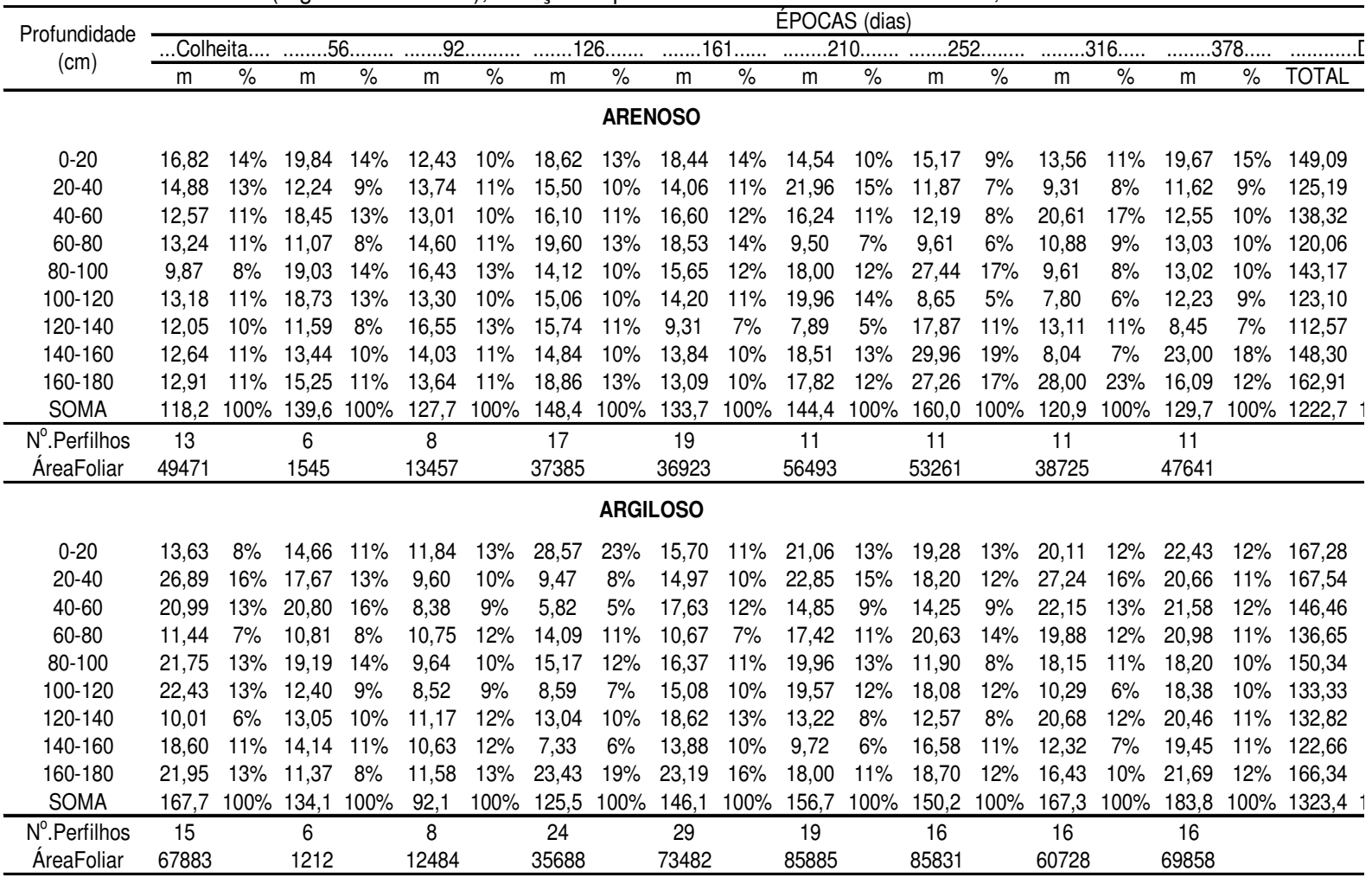

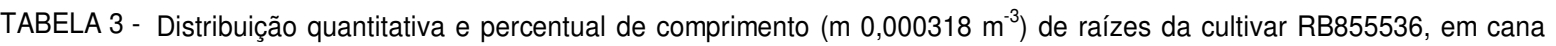
relação à profundidade, em RIZOTRON, através de nove amostragens com cilindro volumétrico, em extratos de substratos (argiloso e arenoso), Estação Experimental de Paranavaí/PR - UFPR, JUL/2001.

\begin{tabular}{|c|c|c|c|c|c|c|c|c|c|c|c|c|c|c|c|c|c|c|c|}
\hline \multirow{3}{*}{$\begin{array}{l}\text { Profundidade } \\
\qquad(\mathrm{cm})\end{array}$} & \multicolumn{19}{|c|}{ ÉPOCAS (dias) } \\
\hline & \multicolumn{2}{|c|}{..Colheita... } & \multicolumn{2}{|c|}{$.56 \ldots$} & \multicolumn{2}{|c|}{.92.} & \multicolumn{2}{|c|}{$.126 .}$. & \multicolumn{2}{|c|}{$.161 \ldots \ldots$} & \multicolumn{2}{|c|}{$\ldots \ldots \ldots 210 \ldots \ldots$} & \multicolumn{2}{|c|}{.252.} & \multicolumn{2}{|c|}{$\ldots \ldots .316 \ldots$} & \multicolumn{2}{|c|}{. $.378 \ldots$} & \multirow[b]{2}{*}{ TOTAL } \\
\hline & $\mathrm{m}$ & $\%$ & $\mathrm{~m}$ & $\%$ & $\mathrm{~m}$ & $\%$ & $\mathrm{~m}$ & $\%$ & $\mathrm{~m}$ & $\%$ & $\mathrm{~m}$ & $\%$ & $\mathrm{~m}$ & $\%$ & $\mathrm{~m}$ & $\%$ & $\mathrm{~m}$ & $\%$ & \\
\hline \multicolumn{20}{|c|}{ ARENOSO } \\
\hline $0-20$ & 13,66 & $10 \%$ & 19,58 & $12 \%$ & 20,75 & $13 \%$ & 22,68 & $11 \%$ & 23,62 & $13 \%$ & 22,68 & $13 \%$ & 21,15 & $12 \%$ & 26,13 & $13 \%$ & 25,53 & $12 \%$ & 195,79 \\
\hline $20-40$ & 12,64 & $9 \%$ & 20,89 & $13 \%$ & 21,77 & $13 \%$ & 24,66 & $12 \%$ & 20,97 & $11 \%$ & 24,08 & $13 \%$ & 17,74 & $10 \%$ & 25,87 & $13 \%$ & 24,22 & $12 \%$ & 192,84 \\
\hline $40-60$ & 15,36 & $11 \%$ & 21,07 & $13 \%$ & 17,04 & $10 \%$ & 25,55 & $13 \%$ & 17,59 & $10 \%$ & 23,26 & $13 \%$ & 21,16 & $12 \%$ & 25,99 & $13 \%$ & 24,55 & $12 \%$ & 191,58 \\
\hline $60-80$ & 11,36 & $8 \%$ & 20,57 & $13 \%$ & 22,76 & $14 \%$ & 25,82 & $13 \%$ & 20,02 & $11 \%$ & 20,14 & $11 \%$ & 21,89 & $12 \%$ & 12,79 & $7 \%$ & 22,68 & $11 \%$ & 178,03 \\
\hline $80-100$ & 12,35 & $9 \%$ & 17,41 & $11 \%$ & 20,83 & $13 \%$ & 22,54 & $11 \%$ & 23,54 & $13 \%$ & 24,30 & $13 \%$ & 20,23 & $11 \%$ & 23,02 & $12 \%$ & 23,68 & $11 \%$ & 187,89 \\
\hline $100-120$ & 16,55 & $12 \%$ & 11,80 & $7 \%$ & 16,99 & $10 \%$ & 25,03 & $13 \%$ & 14,64 & $8 \%$ & 14,15 & $8 \%$ & 22,16 & $12 \%$ & 21,76 & $11 \%$ & 19,79 & $9 \%$ & 162,88 \\
\hline $120-140$ & 13,11 & $9 \%$ & 17,62 & $11 \%$ & 13,52 & $8 \%$ & 18,98 & $10 \%$ & 23,05 & $13 \%$ & 18,74 & $10 \%$ & 21,28 & $12 \%$ & 12,91 & $7 \%$ & 19,24 & $9 \%$ & 158,45 \\
\hline $140-160$ & 28,76 & $20 \%$ & 16,03 & $10 \%$ & 15,93 & $10 \%$ & 16,66 & $8 \%$ & 13,35 & $7 \%$ & 17,53 & $10 \%$ & 17,15 & $10 \%$ & 24,99 & $13 \%$ & 24,29 & $12 \%$ & 174,70 \\
\hline $160-180$ & 18,43 & $13 \%$ & 15,12 & $9 \%$ & 14,36 & $9 \%$ & 15,91 & $8 \%$ & 26,92 & $15 \%$ & 15,44 & $9 \%$ & 16,28 & $9 \%$ & 20,70 & $11 \%$ & 26,48 & $13 \%$ & 169,64 \\
\hline SOMA & 142,2 & $100 \%$ & 160,1 & $100 \%$ & 163,9 & $100 \%$ & 197,8 & $100 \%$ & 183,7 & $100 \%$ & 180,3 & $100 \%$ & 179,0 & $100 \%$ & 194,2 & $100 \%$ & 210,5 & $100 \%$ & 1611,8 \\
\hline $\mathrm{N}^{0}$.Perfilhos & 13 & & 32 & & 26 & & 33 & & 29 & & 17 & & 14 & & 13 & & 13 & & \\
\hline ÁreaFoliar & 38120 & & 4447 & & 24352 & & 38933 & & 36347 & & 52463 & & 45364 & & 20360 & & 33050 & & \\
\hline \multicolumn{20}{|c|}{ ARGILOSO } \\
\hline $0-20$ & 9,29 & $15 \%$ & 12,95 & $11 \%$ & 10,11 & $12 \%$ & 14,34 & $12 \%$ & 21,80 & $17 \%$ & 22,91 & $14 \%$ & 22,10 & $13 \%$ & 19,06 & $11 \%$ & 21,44 & $12 \%$ & 154,00 \\
\hline $20-40$ & 14,87 & $24 \%$ & 14,62 & $12 \%$ & 9,27 & $11 \%$ & 10,59 & $9 \%$ & 12,97 & $10 \%$ & 18,18 & $11 \%$ & 19,29 & $11 \%$ & 21,12 & $12 \%$ & 19,22 & $11 \%$ & 140,13 \\
\hline $40-60$ & 2,70 & $4 \%$ & 15,32 & $13 \%$ & 8,06 & $10 \%$ & 13,11 & $11 \%$ & 19,19 & $15 \%$ & 20,20 & $12 \%$ & 9,87 & $6 \%$ & 12,02 & $7 \%$ & 21,97 & $12 \%$ & 122,46 \\
\hline $60-80$ & 17,44 & $28 \%$ & 12,06 & $10 \%$ & 13,07 & $16 \%$ & 14,26 & $11 \%$ & 9,37 & $7 \%$ & 18,50 & $11 \%$ & 22,92 & $13 \%$ & 26,39 & $15 \%$ & 30,78 & $17 \%$ & 164,77 \\
\hline $80-100$ & 2,34 & $4 \%$ & 12,85 & $11 \%$ & 8,61 & $11 \%$ & 18,42 & $15 \%$ & 10,38 & $8 \%$ & 16,00 & $9 \%$ & 22,90 & $13 \%$ & 10,44 & $6 \%$ & 16,88 & $9 \%$ & 118,81 \\
\hline $100-120$ & 2,51 & $4 \%$ & 14,16 & $12 \%$ & 7,91 & $10 \%$ & 15,42 & $12 \%$ & 9,26 & $7 \%$ & 17,76 & $10 \%$ & 20,68 & $12 \%$ & 11,24 & $6 \%$ & 20,93 & $11 \%$ & 119,88 \\
\hline $120-140$ & 3,62 & $6 \%$ & 8,24 & $7 \%$ & 6,26 & $8 \%$ & 11,29 & $9 \%$ & 13,51 & $11 \%$ & 17,77 & $10 \%$ & 9,89 & $6 \%$ & 29,34 & $16 \%$ & 11,43 & $6 \%$ & 111,35 \\
\hline $140-160$ & 2,67 & $4 \%$ & 13,49 & $11 \%$ & 8,88 & $11 \%$ & 13,60 & $11 \%$ & 12,09 & $10 \%$ & 18,40 & $11 \%$ & 22,57 & $13 \%$ & 17,12 & $10 \%$ & 21,22 & $12 \%$ & 130,03 \\
\hline $160-180$ & 6,59 & $11 \%$ & 14,42 & $12 \%$ & 9,65 & $12 \%$ & 13,38 & $11 \%$ & 16,61 & $13 \%$ & 19,79 & $12 \%$ & 24,80 & $14 \%$ & 31,39 & $18 \%$ & 18,77 & $10 \%$ & 155,40 \\
\hline SOMA & 62,03 & $100 \%$ & 118,1 & $100 \%$ & 81,81 & $100 \%$ & 124,4 & $100 \%$ & 125,2 & $100 \%$ & 169,5 & $100 \%$ & 175,0 & $100 \%$ & 178,1 & $100 \%$ & 182,6 & $100 \%$ & 1216,8 \\
\hline $\mathrm{N}^{0}$.Perfilhos & 15 & & 24 & & 30 & & 40 & & 35 & & 18 & & 14 & & 14 & & 14 & & \\
\hline ÁreaFoliar & 41839 & & 2690 & & 28050 & & 53704 & & 52918 & & 64417 & & 61257 & & 35286 & & 44475 & & \\
\hline
\end{tabular}


IDO, O.T. et al. Crescimento e distribuição radical...

TABELA 4 - Distribuição quantitativa e percentual de comprimento $\left(\mathrm{m} \mathrm{0,000318} \mathrm{m}^{-3}\right)$ de raízes da cultivar SP80-1842, em cana relação à profundidade, em RIZOTRON, através de nove amostragens com cilindro volumétrico, em extratos de substratos (argiloso e arenoso), Estação Experimental de Paranavaí/PR - UFPR, JUL/2001.

\begin{tabular}{|c|c|c|c|c|c|c|c|c|c|c|c|c|c|c|c|c|c|c|c|}
\hline \multirow{3}{*}{$\begin{array}{l}\text { Profundidade } \\
\text { (cm) }\end{array}$} & \multicolumn{19}{|c|}{ EPOCAS (dias) } \\
\hline & \multicolumn{2}{|c|}{. Colheita.... } & \multicolumn{2}{|c|}{$.56 \ldots \ldots \ldots$} & \multicolumn{2}{|c|}{$.92 \ldots$} & \multicolumn{2}{|c|}{$\ldots \ldots 126 \ldots \ldots$} & \multicolumn{2}{|c|}{$\ldots \ldots .161 \ldots \ldots$} & \multicolumn{2}{|c|}{........210 } & \multicolumn{2}{|c|}{$\ldots 252 .}$. & \multicolumn{2}{|c|}{........316.... } & \multicolumn{2}{|c|}{.......378..... } & \multirow{2}{*}{ TOTAL } \\
\hline & $\mathrm{m}$ & $\%$ & $\mathrm{~m}$ & $\%$ & $\mathrm{~m}$ & $\%$ & $\mathrm{~m}$ & $\%$ & $\mathrm{~m}$ & $\%$ & $m$ & $\%$ & $\mathrm{~m}$ & $\%$ & $m$ & $\%$ & $\mathrm{~m}$ & $\%$ & \\
\hline \multicolumn{20}{|c|}{ ARENOSO } \\
\hline $0-20$ & 15,08 & $15 \%$ & 18,01 & $12 \%$ & 11,62 & $9 \%$ & 17,37 & $11 \%$ & 20,29 & $16 \%$ & 18,26 & $14 \%$ & 16,30 & $12 \%$ & 14,65 & $12 \%$ & 21,62 & $15 \%$ & 153,21 \\
\hline $20-40$ & 9,07 & $9 \%$ & 15,60 & $10 \%$ & 17,13 & $14 \%$ & 14,95 & $9 \%$ & 13,11 & $10 \%$ & 20,05 & $15 \%$ & 9,92 & $7 \%$ & 13,32 & $11 \%$ & 24,78 & $17 \%$ & 137,94 \\
\hline $40-60$ & 9,32 & $9 \%$ & 18,62 & $12 \%$ & 16,50 & $13 \%$ & 18,17 & $12 \%$ & 15,04 & $12 \%$ & 14,13 & $11 \%$ & 16,53 & $12 \%$ & 13,71 & $11 \%$ & 15,60 & $11 \%$ & 137,62 \\
\hline $60-80$ & 6,01 & $6 \%$ & 15,12 & $10 \%$ & 14,47 & $11 \%$ & 20,38 & $13 \%$ & 13,12 & $10 \%$ & 8,15 & $6 \%$ & 10,95 & $8 \%$ & 18,76 & $15 \%$ & 14,46 & $10 \%$ & 121,42 \\
\hline $80-100$ & 12,95 & $12 \%$ & 10,52 & $7 \%$ & 12,84 & $10 \%$ & 21,89 & $14 \%$ & 17,03 & $13 \%$ & 11,64 & $9 \%$ & 20,95 & $16 \%$ & 9,65 & $8 \%$ & 10,57 & $7 \%$ & 128,04 \\
\hline $100-120$ & 10,09 & $10 \%$ & 17,69 & $12 \%$ & 14,26 & $11 \%$ & 13,97 & $9 \%$ & 10,91 & $8 \%$ & 18,23 & $14 \%$ & 13,44 & $10 \%$ & 10,21 & $8 \%$ & 10,80 & $7 \%$ & 119,61 \\
\hline $120-140$ & 23,60 & $23 \%$ & 18,87 & $13 \%$ & 11,90 & $9 \%$ & 18,93 & $12 \%$ & 12,05 & $9 \%$ & 14,27 & $11 \%$ & 17,46 & $13 \%$ & 12,44 & $10 \%$ & 14,47 & $10 \%$ & 143,98 \\
\hline $140-160$ & 8,76 & $8 \%$ & 17,22 & $11 \%$ & 16,15 & $13 \%$ & 16,50 & $10 \%$ & 11,43 & $9 \%$ & 8,94 & $7 \%$ & 10,18 & $8 \%$ & 13,71 & $11 \%$ & 14,03 & $9 \%$ & 116,91 \\
\hline $160-180$ & 8,83 & $9 \%$ & 18,97 & $13 \%$ & 11,93 & $9 \%$ & 15,68 & $10 \%$ & 15,66 & $12 \%$ & 16,85 & $13 \%$ & 18,46 & $14 \%$ & 18,21 & $15 \%$ & 22,22 & $15 \%$ & 146,81 \\
\hline SOMA & 103,7 & $100 \%$ & 150,6 & $100 \%$ & 126,8 & $100 \%$ & 157,8 & $100 \%$ & 128,6 & $100 \%$ & 130,5 & $100 \%$ & 134,2 & $100 \%$ & 124,7 & $100 \%$ & 148,6 & $100 \%$ & 1205,5 \\
\hline $\mathrm{N}^{0}$.Perfilhos & 13 & & 11 & & 15 & & 24 & & 17 & & 13 & & 11 & & 11 & & 11 & & \\
\hline ÁreaFoliar & 45494 & & 1681 & & 22637 & & 36965 & & 53873 & & 56981 & & 45731 & & 35920 & & 31658 & & \\
\hline \multicolumn{20}{|c|}{ ARGILOSO } \\
\hline $0-20$ & 21,16 & $15 \%$ & 27,33 & $19 \%$ & 17,68 & $18 \%$ & 18,68 & $12 \%$ & 20,96 & $14 \%$ & 12,14 & $8 \%$ & 34,21 & $24 \%$ & 29,77 & $13 \%$ & 24,47 & $12 \%$ & 206,39 \\
\hline $20-40$ & 17,13 & $12 \%$ & 6,84 & $5 \%$ & 8,81 & $9 \%$ & 17,73 & $11 \%$ & 19,31 & $13 \%$ & 20,15 & $14 \%$ & 28,74 & $20 \%$ & 21,61 & $9 \%$ & 25,52 & $12 \%$ & 165,85 \\
\hline $40-60$ & 24,07 & $18 \%$ & 22,15 & $16 \%$ & 12,08 & $13 \%$ & 18,09 & $11 \%$ & 20,28 & $13 \%$ & 16,79 & $11 \%$ & 12,21 & $8 \%$ & 28,25 & $12 \%$ & 20,98 & $10 \%$ & 174,90 \\
\hline $60-80$ & 15,57 & $11 \%$ & 23,76 & $17 \%$ & 9,19 & $10 \%$ & 18,27 & $12 \%$ & 18,05 & $12 \%$ & 17,23 & $12 \%$ & 18,22 & $13 \%$ & 25,92 & $11 \%$ & 24,10 & $12 \%$ & 170,32 \\
\hline $80-100$ & 26,94 & $20 \%$ & 16,50 & $12 \%$ & 10,90 & $11 \%$ & 17,21 & $11 \%$ & 10,25 & $7 \%$ & 14,62 & $10 \%$ & 11,28 & $8 \%$ & 30,50 & $13 \%$ & 27,52 & $13 \%$ & 165,72 \\
\hline $100-120$ & 4,53 & $3 \%$ & 4,52 & $3 \%$ & 6,49 & $7 \%$ & 18,85 & $12 \%$ & 13,48 & $9 \%$ & 18,38 & $12 \%$ & 15,75 & $11 \%$ & 32,29 & $14 \%$ & 16,10 & $8 \%$ & 130,39 \\
\hline $120-140$ & 16,22 & $12 \%$ & 13,12 & $9 \%$ & 9,70 & $10 \%$ & 12,26 & $8 \%$ & 13,28 & $9 \%$ & 23,54 & $16 \%$ & 10,43 & $7 \%$ & 23,70 & $10 \%$ & 26,82 & $13 \%$ & 149,07 \\
\hline $140-160$ & 7,01 & $5 \%$ & 7,76 & $5 \%$ & 7,65 & $8 \%$ & 17,90 & $11 \%$ & 15,31 & $10 \%$ & 12,72 & $9 \%$ & 6,50 & $5 \%$ & 24,99 & $11 \%$ & 19,43 & $9 \%$ & 119,27 \\
\hline $160-180$ & 4,86 & $4 \%$ & 19,94 & $14 \%$ & 14,11 & $15 \%$ & 18,97 & $12 \%$ & 20,35 & $13 \%$ & 12,69 & $9 \%$ & 6,53 & $5 \%$ & 11,22 & $5 \%$ & 22,53 & $11 \%$ & 131,20 \\
\hline SOMA & 137,5 & $100 \%$ & 141,9 & $100 \%$ & 96,61 & $100 \%$ & 158,0 & $100 \%$ & 151,3 & $100 \%$ & 148,3 & $100 \%$ & 143,9 & $100 \%$ & 228,3 & $100 \%$ & 207,5 & $100 \%$ & 1413,1 \\
\hline $\mathrm{N}^{\circ}$.Perfilhos & 11 & & 15 & & 14 & & 29 & & 21 & & 14 & & 13 & & 13 & & 13 & & \\
\hline ÁreaFoliar & 32628 & & 2195 & & 17285 & & 43523 & & 53101 & & 68213 & & 58515 & & 38129 & & 40510 & & \\
\hline
\end{tabular}

Observam-se nas Tabelas 2, 3, 4, independente da cultivar e do tipo de solo, na fase inicial de desenvolvimento da cana de primeira soca até 56 DAC (período de aumento na brotação de perfilhos), aumento na produção inicial das raízes (comprimento), distribuindo ao longo do perfil dos substratos, com leve tendência de concentração das raízes nas camadas mais superficiais de 0-60 cm, em função de que após o corte da cana planta, o sistema antigo mantém-se ainda em atividade por algum tempo, quando tem início a sua substituição pelas raízes dos novos perfilhos da cana soca. Cada perfilho apresenta um sistema radicial próprio, de forma que, enquanto houver emissão de perfilhos, ocorre aumento no volume de raízes (BACCHI, 1985; CASTRO, 2001).

Por volta dos 92 DAC, verifica-se tendência geral de decréscimo no crescimento de raízes, principalmente nas camadas intermediárias do perfil dos substratos $(60-140 \mathrm{~cm})$, como suposição, talvez em função da desativação ou morte do sistema radicial da cana planta. Durante o período de intenso perfilhamento (92 a 161 DAC) ocorre novamente um aumento no comprimento das raízes. Após o período de máximo perfilhamento (160 até 210 DAC), sucede decréscimo na produção radicial, manifestada principalmente, nas camadas intermediárias de 100 a $160 \mathrm{~cm}$, coincidindo como o período de diminuição no número de colmos e área foliar máxima, o que segundo MIOCQUE (1999) é uma das épocas de máximo crescimento aéreo. AGUIAR (1978) demonstrou que a cana não forma um sistema radicial definitivo, há alternância de raízes vivas e mortas durante o ciclo da cultura.

Por volta dos 210 a 252 DAC, conforme a cultivar, continua a fase de decréscimo na produção de raízes, período coincidente com a diminuição e estabilização do número de colmos finais, que pode ser explicado, talvez, em função da morte do sistema radicial respectivo dos perfilhos perecidos, somado com a morte das raízes da cana planta em camadas intermediárias, o que pode ser verificado em função da maior porcentagem de diminuição ocorrer nas camadas mais superficiais e intermediárias. Do período de estabilização no número final de colmos (252 DAC) até o momento da colheita (378 DAC), ocorre tendência de aumento e estabilização na produção de raízes.

\section{CONCLUSÕES}

Os resultados obtidos nas condições em que o experimento foi conduzido permitem as seguintes conclusões:

- O rizotron é uma estrutura viável para o estudo do sistema radicial da cana de primeira soca;

- O substrato influenciou o crescimento radicial das cultivares, em cana de primeira soca;

- As cultivares RB835486 e SP80-1842, apresentaram maiores comprimentos totais de raízes no substrato argiloso, enquanto a cultivar RB85536 mostrou maiores valores no sistema radicial no substrato arenoso. 
IDO, O.T. et al. Crescimento e distribuição radical...

\section{REFERÊNCIAS}

1. AGUIAR, S.F. Observações sobre sistema radicular de cana planta (Saccharum spp.). Jaboticabal: 1978. 24p. Trabalho de Graduação (Graduação em Agronomia - Faculdade de Ciências Agrárias e Veterinárias, Campus Jaboticabal, UNESP).

2. ARIZONO, H.; GHELLER, A.C.A.; MASUDA, Y.; HOFMANN, H.P.; BASSINELLO, A.I.; MATSUOKA, S.; GIGLIOT, E.A.; MENEZES, L.L. Guia das principais variedades de cana-de-açúcar RB 2000. Araras: UFSCar, 2000. 16p.

3. BACCHI, O.O.S. Ecofisiologia da cana-de-açúcar. Piracicaba: IAA/Planalsucar, 1985. 20p.

4. BÖHM, W. Methods of studying root systems. Berlin: Springler-Verlag, 1979. 188p.

5. CASTRO, P.R.C. Utilização de reguladores vegetais no sistema de produção da cana-de-açúcar. In: SIMPÓSIO INTERNACIONAL DE FISIOLOGIA DA CANA-DE-AÇÚCAR, 2000, Piracicaba. Anais... Piracicaba, STAB, 20011 CD-ROM

6. DAROS, E.; ZAMBON, J.L.C; WEBER, H.; IDO, O.T.; GRACIANO, P.A.; ZANETTE, F. Desenvolvimento e distribuição de raízes de diferentes culturas, em condições de rizotron, no Paraná. In: WORKSHOP SOBRE SISTEMARADICULAR: METODOLOGIAS E ESTUDO DE CASOS, 1999, Aracaju. Anais... Aracaju: Embrapa Tabuleiros Costeiros, p.167-178, 1999.

7. EMBRAPA - Centro Nacional de Pesquisa de Solos (Rio de Janeiro, RJ.) Sistema brasileiro de classificação de solos. Brasília: Embrapa Produções de Informações; Rio de Janeiro: Embrapa Solos, 1999, 412p.

8. JORGE, L.A.C. Descrição detalhada de trincheira com produção de imagem para uso do SIARCS®. In: WORKSHOP SOBRE SISTEMA RADICULAR: METODOLOGIAS E ESTUDO DE CASOS, 1999, Aracaju. Anais... Aracaju: Embrapa Tabuleiros Costeiros, p.255-268, 1999.

9. KORNDÓRFER, G.H., PRIMAVESI, O., DEUBER, R. Crescimento e distribuição do sistema radicular da cana-de-açúcar em solo LVA. Boletim Técnico Copersucar, Piracicaba, v.47, p.32-36, 1989.

10. MATSUOKA, S. Botânica e ecofisiologia da cana-de-açúcar. In: CURSO DE QUALIFICAÇÃO EM PLANTAS INDUSTRIAIS - Cana-de-açúcar. Maringá: UFPR/SENAR, 1996. 34p. (Apostila).

11. MATTA, F.M. Mecanismos fisiológicos associados ao desenvolvimento do sistema radicular das plantas. In: WORKSHOP SOBRE SISTEMA RADICULAR: METODOLOGIAS E ESTUDO DE CASOS, 1999, Aracaju. Anais... Aracaju: Embrapa Tabuleiros Costeiros, p.19-45, 1999.

12. MIOCQUE, J. Avaliação de crescimento e de produtividade de matéria verde da cana-de-açúcar na região de Araraquara - SP. STAB, Piracicaba, v.17, n.4, p.45-47, 1999.

13. RÉGENT INSTRUMENTS. Win/MacRHIZO V4.1c Introduction manual. Régent Instruments Inc., Québec, Canada. 36p., 1999a.

14. RÉGENT INSTRUMENTS. Win/MacRHIZO V4.1c Reference. Régent Instruments Inc., Québec, Canada. 51p., 1999b.

15. TAYLOR, H.M. Managing root systems to reduce plant water deficits. In: RUSSEL, R.S.; IGUE, K.; MEHTA, Y.R. (Ed.) THE SOIL/ROOT SYSTEM IN RELATION TO BRAZILAGRICULTURE. 1980, Londrina. Proceedings... Londrina: Fundação Instituto Agronômico do Paraná, p.45-60, 1981.

16. VASCONCELOS, A.C.M.; CASAGRANDE, A.A.; LANDELL, M.G.A.; BARBOSA, J.C.; DORIZOTTO, P.H.; FOGAÇA, J.F. Desenvolvimento do sistema radicular e produtividades agroindustriais de cana-de-açúcar no Vale do Paranapanema. In: CONGRESSO NACIONAL DA STAB, 7., Londrina, 1999. Anais... São Paulo, STAB, p.78-81, 1999.

17. VASCONCELOS, A.C.M. O sistema radicular da cana-de-açúcar e a expressão do potencial de produção. STAB, Piracicaba, v.21, n.2, p.20, 2002.

18. VITTI, G.A.; MAZZA, J.A. Planejamento, estratégias de manejo e nutrição da cultura de cana-de-açúcar. Piracicaba: POTAFOS, 2002. 16p. (Informações Agronômicas, 97)

19. WEI, C.C., YANG, P.C. Investigations of sugar cane root distribution of two different varieties in the rhizotron. Taiwan Sugar., v.34, n.2, p.8-11. 1987.

Recebido em 16/07/2003 Aceito em 19/09/2006 